\title{
Simple Cost Sharing Schemes for Multicommodity Rent-or-Buy and Stochastic Steiner Tree
}

\author{
Lisa Fleischer ${ }^{*}$
}

Jochen Könemann $^{\dagger}$

\begin{abstract}
In the multi-commodity rent-or-buy network design problem (MRoB) we are given a network together with a set of $k$ terminal pairs $R=\left\{\left(s_{1}, t_{1}\right), \ldots,\left(s_{k}, t_{k}\right)\right\}$. The goal is to install capacities on the edges of the network so that a prescribed amount of flow $f_{i}$ can be routed between all terminal pairs $s_{i}$ and $t_{i}$ simultaneously. We can either rent capacity on an edge at some cost per unit flow or buy infinite capacity on an edge at some larger fixed cost. The overall objective is to install capacities at a minimum total cost.

The version of the stochastic Steiner tree problem (SST) considered here is the Steiner tree problem in the model of two-stage stochastic optimization with recourse. In stage one, there is a known probability distribution on subsets of vertices and we can choose to buy a subset of edges at a given cost. In stage two, a subset of vertices $T$ from the prior known distribution is realized, and additional edges can be bought at a possibly higher cost. The objective is to buy a set of edges in stages one and two so that all vertices in $T$ are connected, and the expected cost is minimized.

Gupta et al. (FOCS '03) give a randomized scheme for the MRoB problem that was both used subsequently to improve the approximation ratio for this problem, and extended to yield the best approximation algorithm for SST. One building block of this scheme is a good approximation algorithm for Steiner forests.
\end{abstract}

\footnotetext{
* Supported in part by NSF grant CCF-0515127. P. O. Box 218, T. J. Watson Research Ctr., IBM, Yorktown Heights, NY 10598, USA.Email: 1kf@watson.ibm.com.

tDepartment of Combinatorics and Optimization, University of Waterloo, 200 University Avenue West, Waterloo, ON N2L 3G1, Canada. Supported by NSERC grant no. 288340-2004 and by an IBM Faculty Award. Email: jochen@uwaterloo.ca.

†Dipartimento di Informatica e Sistemistica, University of Rome "La Sapienza", Via Salaria 113, 00198 Rome, Italy. Part of this work was done while the author was visiting the School of Computer Science at Carnegie Mellon University. Email: leon@dis.uniroma1.it.

$\S_{\text {Supported by the DFG Research Center Matheon "Mathematics }}$ for key technologies". Institute for Mathematics, Technical University Berlin, Straße des 17. Juni 136, 10623 Berlin, Germany. Email: schaefer@math.tu-berlin.de.
}

Permission to make digital or hard copies of all or part of this work for personal or classroom use is granted without fee provided that copies are not made or distributed for profit or commercial advantage and that copies bear this notice and the full citation on the first page. To copy otherwise, to republish, to post on servers or to redistribute to lists, requires prior specific permission and/or a fee.

STOC'06, May21-23, 2006, Seattle, Washington, USA

Copyright 2006 ACM 1-59593-134-1/06/0005 ...\$5.00.

\author{
Stefano Leonardi $\quad$ Guido Schäfer ${ }^{\ddagger}$
}

\begin{abstract}
We present a surprisingly simple 5-approximation algorithm for MRoB and 6-approximation for SST, improving on the best previous guarantees of 6.828 and 12.6, and show that no approximation ratio better than 4.67 can be achieved using the above mentioned randomized scheme in combination with the currently best known Steiner forest approximation algorithms. A key component of our approach are cost shares that are 3-strict for the unmodified primaldual Steiner forest algorithm.
\end{abstract}

\section{Categories and Subject Descriptors}

F.2.2 [Analysis of Algorithms and Problem Complexity]: Nonnumerical Algorithms and Problems

\section{General Terms}

Algorithms, Design, Theory

\section{Keywords}

approximation algorithms, stochastic optimization, cost sharing

\section{INTRODUCTION}

Multicommodity Rent-or-Buy. In the multi-commodity rentor-buy problem (MRoB) we are given an undirected graph $G=$ $(V, E)$, terminal pairs $R=\left\{\left(s_{1}, t_{1}\right), \ldots,\left(s_{k}, t_{k}\right)\right\} \subseteq V \times V$, nonnegative costs $c_{e}$ for all edges $e \in E$, and a parameter $M \geq 0$. The goal is to install capacities on the edges of $G$ such that for all $\left(s_{i}, t_{i}\right) \in R$ we can simultaneously route a given amount of flow $f_{i}$ from $s_{i}$ to $t_{i}$. We can either rent capacity on an edge $e$ at cost $\lambda(e) \cdot c(e)$, where $\lambda(e)$ is the flow traversing edge $e$, or buy infinite capacity on edge $e$ at cost $M \cdot c(e)$. Bought edges have no incremental, flow-dependent cost. The overall objective is to find a feasible solution of smallest total cost.

The MRoB problem generalizes a number of fundamental optimization problems. For $M=\infty$, an optimum solution for an $\mathrm{MRoB}$ instance can be found by connecting each pair of terminals by their shortest path.

For $M=1$, MRoB reduces to the Steiner forest problem. The Steiner forest problem is to compute a minimum-cost forest that contains an $s_{i}, t_{i}$-path for all $1 \leq i \leq k$. It is well-known that this problem is NP-hard [9] and even Max-SNP hard [7]. The best known approximation algorithm achieves a performance guarantee of $2-1 / k$ and is due to Agrawal, Klein and Ravi [3]. Goemans and Williamson [15] generalize these results to a larger class of network design problems.

The MRoB problem is a generalization of the single-commodity rent-or-buy problem ( $\mathrm{SRoB})$. Here, in addition to the input given in an instance of the MRoB problem, one also has a root node $r \in V$. 
The root $r$ is part of every terminal pair, i.e., $r \in\left\{s_{i}, t_{i}\right\}$ for all $1 \leq i \leq k$. Gupta et al. [11] gave a randomized 3.55-approximation algorithm for the problem.

Kumar, Gupta and Roughgarden [16] give the first constantfactor approximation algorithm for the MRoB problem. Based on the techniques used by Gupta et al. [11] for the single-commodity rent-or-buy problem, Gupta et al. [10] present a 12-approximation algorithm for the MRoB problem. Becchetti et al. [6] recently obtained the currently best known 6.828-approximation algorithm for this problem.

The MRoB problem is a special case of the multicommodity buyat-bulk $(\mathrm{MBaB})$ problem. The input in this problem is as in the MRoB problem, except for an additional sub-additive monotone function $l: \mathbb{Z}^{+} \rightarrow \mathbb{R}^{+}$. A feasible solution consists of a vector $x \in$ $\mathbb{Z}_{|E|}^{+}$of edge-capacities that allows for $f_{i}$ units of flow to be routed between $s_{i}$ and $t_{i}$, for all $\left(s_{i}, t_{i}\right) \in R$ simultaneously, and feasibly. The cost of installing capacities $x$ is $\sum_{e \in E} l\left(x_{e}\right) c_{e}$ and the goal is to find a feasible capacity installation $x$ of minimum total cost.

In [2], Awerbuch and Azar present an $O(\alpha)$-approximation for $\mathrm{MBaB}$, assuming that any metric can be probabilistically approximated by a family of tree metrics with an expected distortion at most $\alpha$. In [4], Bartal shows $\alpha=O\left(\log ^{2} n\right)$ and improves this bound in [5] to $\alpha=O(\log n \log \log n)$. More recently, Fakcharoenphol et al. [8] show that $\alpha=O(\log n)$. Recently, Andrews [1] shows that the results in [2] and [8] are best possible up to constant factors unless NP $\subseteq$ ZPTIME $\left(n^{\text {polylog }(n)}\right)$.

Stochastic Steiner Tree. The stochastic Steiner tree problem (SST) we consider here is the Steiner tree problem in the model of two-stage stochastic optimization with recourse. In stage one, there is a known probability distribution $\pi$ on subsets of vertices and we can chose to buy a subset of edges at a given cost. In stage two, a subset of vertices $T$ from the prior known distribution is realized, and additional edges can be bought at a possibly higher cost. The objective is to buy a set of edges in stages one and two so that all vertices in $T$ are connected, and the expected cost is minimized.

We make no assumptions about the distribution $\pi$ on the subset of vertices, except that we have access to it via a sampling oracle: on request, the oracle outputs a subset of vertices $T$ drawn from the distribution. Gupta and Pál give a 12.6-approximation for SST [12]. Prior to their work, there were constant factor guarantees for the problem when all of the possible subsets realized in stage two contain a fixed root terminal $[13,14]$.

Common Framework. Our work uses a common framework developed in Gupta et al. [10] for MRoB and extended by Gupta and Pál in [12] for SST. This framework first chooses a random subset $S \subseteq R$ of the set of terminal pairs. It then computes an approximate Steiner forest $F_{S}$ for $S$ using an adaptation of the primal-dual algorithm for Steiner forests and buys its edges. Finally, this forest is augmented to a feasible solution for $R$ by renting additional edges in a cheapest possible way such that all remaining terminals in $R \backslash S$ are connected.

The performance of the above framework depends strongly on a certain stability property of the Steiner forest algorithm used to compute $F_{S}$. For a forest $F$ in $G$, let $G \mid F$ denote the graph resulting from contracting all trees of $F$. We use $c_{G \mid F}(u, v)$ to denote the minimum cost of any $u, v$-path in $G \mid F$. For a parameter $\beta>0$, Gupta et al. [10] define the notion of $\beta$-strict algorithms for the minimum-cost Steiner forest problem:

DEFINITION 1. An algorithm ALG for the Steiner forest problem is $\beta$-strict if there exist cost shares $\xi_{\text {st }}$ for all $(s, t) \in R$ such that

1. $\sum_{(s, t) \in R} \xi_{s t} \leq \mathrm{opt}_{R}$, where $\mathrm{opt}_{R}$ is the minimum cost of $a$

\section{Steiner forest for $R$, and}

2. $c_{G \mid F_{-s t}}(s, t) \leq \beta \cdot \xi_{s t}$ for all $(s, t) \in R$, where $F_{-s t}$ is a Steiner forest for terminal set $R_{-s t}=R \backslash\{(s, t)\}$ returned by ALG.

Gupta et al. [10] then show that using an $\alpha$-approximate and $\beta$ strict Steiner forest algorithm in their framework yields an $(\alpha+\beta)$ approximation algorithm for the MRoB problem. The authors devise a 6-approximate and 6-strict algorithm for Steiner forests which yields a 12-approximate algorithm for MRoB. Their analysis can be tightened to achieve an 8-approximation. Becchetti et al. [6] reduced the approximation ratio to 6.828 by devising a $(2+\sqrt{2})$-approximate and $(2+\sqrt{2})$-strict primal-dual Steiner forest algorithm.

The notion of strictness defined above assumes that $R$ is a set of terminal pairs. To extend this framework to handle SST, Gupta and Pál extend the notion of strictness to a set $R$ of terminal subsets of arbitrary size, called groups. For a group $g \in R$, let $c_{G \mid F}(g)$ denote the minimum cost of connecting all terminals of $g$ in $G \mid F$.

DEFINITION 2. An algorithm ALG for the Steiner forest problem is $\beta$-group-strict if there exist cost shares $\xi_{g}$ for all $g \in R$ such that

\section{1. $\sum_{g \in R} \xi_{g} \leq \mathrm{opt}_{R}$, where $\mathrm{opt}_{R}$ is the minimum cost of a Steiner} forest for $R$, and

2. $c_{G \mid F_{-g}}(g) \leq \beta \cdot \xi_{g}$ for all $g \in R$, where $F_{-g}$ is a Steiner forest for terminal set $R_{-g}=R \backslash\{g\}$ returned by ALG.

The algorithms in [6], [10], and [12] all adapt the primal-dual Steiner forest algorithm from [3]. In these papers, strictness is achieved by adding extra edges into the Steiner forest produced by the standard primal-dual algorithm. This worsens the approximation ratio but reduces the cost of augmenting a feasible forest $F_{-g}$ into a feasible forest for $R$.

Our Results. In this paper, we show that the primal-dual algorithms for Steiner forest [3,15] are 3-strict and 4-group-strict with appropriate cost sharing rules. We summarize our main contribution in the following theorem:

THEOREM 1. There exists a primal-dual 2-approximate algorithm for the Steiner forest problem that is 3-strict and 4-groupstrict.

This implies a 5-approximation for MRoB and a 6approximation for SST using the framework in $[10,12]$. Moreover, this also implies a 5-approximation algorithm for the 2-stage stochastic Steiner forest problem in the independent decisions model [13].

This is the first algorithm to show that the unmodified primaldual Steiner forest algorithm has constant strict or group-strict cost shares. Finally, we present an example instance that shows that the natural primal-dual Steiner forest algorithm is not $\left(\frac{8}{3}-\varepsilon\right)$-strict for any $\varepsilon>0$, therefore showing that the two-stage analysis of Gupta et al. given in [10] is nearly tight for MRoB.

Outline of Paper. In Section 2, we define the structure of our cost shares and give a surprisingly simple property that implies $2 \alpha$ group-strictness if the cost shares are based on an $\alpha$-approximate Steiner forest. In Section 2, we also present our improved $\frac{3}{2} \alpha$ strictness result for cost shares that satisfy an additional requirement. In Section 3, we review the Steiner forest algorithm AKR and show how the cost of every edge of the forest that is computed is shared between terminal pairs in order to meet the requirements of our strictness results. Finally, in Section 4, we give examples that demonstrate that our results are nearly tight for the framework proposed by [10]. 


\section{STRICTNESS OF COST SHARING AL- GORITHMS FOR STEINER FORESTS}

Suppose we are given an $\alpha$-approximation algorithm ALG that computes a Steiner forest $F$ for the set of terminal groups $R$. In this section, we define two different cost sharing schemes to distribute a fraction of $\frac{1}{\alpha}$ of the cost of $F$ among the terminals. These schemes crucially rely on a notion of witnesses that are associated with each edge $e \in F$. We show that if ALG and the witness definition satisfy certain properties, these cost sharing schemes yield $2 \alpha$-group-strict and $\frac{3}{2} \alpha$-strict cost shares.

We assume without loss of generality that the terminal sets of two different groups in $R$ are disjoint. If $s$ appears in two groups, $g_{1}$ and $g_{2}$, we can create two new nodes $s_{1}$ and $s_{2}$, add edges $\left(s_{1}, s\right)$ and $\left(s_{2}, s\right)$ each of zero cost, and replace $s$ with $s_{1}$ in $g_{1}$ and with $s_{2}$ in $g_{2}$.

Given that $F$ is produced by an $\alpha$-approximation algorithm ALG, we define the cost share $\xi(e)$ of an edge $e \in F$ as

$$
\xi(e)=\frac{1}{\alpha} c(e)
$$

For each edge $e \in F$, we assign two terminals $\mathscr{W}_{e}=\{u, v\}$ to be the witnesses of $e$ and split $\xi(e)$ between the terminals in $\mathscr{W}_{e}$. In this section, we give two different ways of splitting this cost share, yielding two different strictness results.

Let $\xi_{u}(e)$ be the share of $\xi(e)$ of terminal $u \in \mathscr{W}_{e}$ according to the split. The total cost share assigned to terminal $u$ is

$$
\xi_{u}=\sum_{e \in F: u \in \mathscr{W}_{e}} \xi_{u}(e)
$$

The cost share of a group of terminals $g \in R$ is $\xi_{g}=\sum_{u \in g} \xi_{u}$.

We prove that if cost shares are distributed as described above, the total cost share of all groups of terminals does not exceed the optimum cost. This validates condition (1) of Definition 2.

LEMMA 1. Let $F$ be a Steiner forest computed by an $\alpha$ approximate algorithm ALG and let $\left\{\mathscr{W}_{e}\right\}_{e \in F}$ be the associated witness set. If the cost shares $\xi$ are computed as described above then

$$
\sum_{g \in R} \xi_{g} \leq \mathrm{opt}_{R}
$$

Proof. By our cost sharing rule (1), we have

$$
c(F)=\sum_{e \in F} c(e)=\sum_{e \in F} \sum_{u \in \mathscr{W}_{e}} \alpha \xi_{u}(e)=\alpha \sum_{g \in R} \xi_{g}
$$

and this implies the lemma as $c(F) \leq \alpha \cdot \mathrm{opt}_{R}$.

\subsection{Symmetric Cost Share Assignment}

Crucial to proving the strictness of our cost sharing scheme is to define the witness set $\left\{\mathscr{W}_{e}\right\}_{e \in F}$ to satisfy the following property. For a group of vertices $g \in R$, let $T_{g}$ denote the unique tree connecting $g$ in $F$ if such a tree exists; otherwise $T_{g}=\emptyset$. In the following we abuse notation by letting a path $P$ or tree $T$ also stand for the set of edges in it.

PROPERTY 1. Consider an arbitrary group of terminals $g \in R$ and let $e$ be an edge in tree $T_{g}$. If $\mathscr{W}_{e} \cap g=\emptyset$ then $e$ is part of the forest $F_{-g}$.

Remove terminal group $g$ from $R$ and run ALG on the set of terminal groups $R_{-g}=R \backslash\{g\}$. Property 1 implies that if an edge $e \in T_{g}$ is not part of the forest $F_{-g}$ then $e$ is witnessed by some terminal in $g$, i.e., $\mathscr{W}_{e} \cap g \neq \emptyset$.
A natural idea is to split the cost share $\xi(e)$ of $e$ evenly among the two witnesses. This is what we call the symmetric cost share assignment:

Symmetric cost share assignment: The cost share that each witness $u \in \mathscr{W}_{e}$ receives for edge $e$ is

$$
\xi_{u}(e)=\frac{1}{2} \xi(e) .
$$

It is easy to see that Property 1 together with the symmetric cost share assignment yields cost shares that are $2 \alpha$-strict.

Lemma 2. Let $F$ be a Steiner forest computed by an $\alpha$ approximate algorithm ALG and let $\left\{\mathscr{W}_{e}\right\}_{e \in F}$ be the witness set. If algorithm ALG and $\left\{\mathscr{W}_{e}\right\}_{e \in F}$ satisfy Property 1, then the symmetric cost shares $\xi$ are $\beta=2 \alpha$-strict, i.e., for all $g \in R$

$$
c_{G \mid F_{-g}}(g) \leq 2 \alpha \cdot \xi_{g} .
$$

PROOF. Property 1 ensures that all edges $e \in T_{g}$ that are not part of $F_{-g}$ must be witnessed by some terminal in $g$. The claim of the lemma follows since for each edge $e$ of $T_{g}$ with $\mathscr{W}_{e} \cap g \neq \emptyset$, there is some terminal $u$ in $g$ with $\xi_{u}(e)=\frac{1}{2} \xi(e)=\frac{1}{2 \alpha} c(e)$.

\subsection{Asymmetric Cost Share Assignment}

We next turn to a refined asymmetric cost sharing scheme, where we split the cost share $\xi(e)$ of an edge $e \in F$ unevenly among its witnesses in $\mathscr{W}_{e}$. We prove that this asymmetric cost sharing scheme yields $\frac{3}{2} \alpha$-strict cost shares, if only algorithm ALG and the witness set $\left\{\mathscr{W}_{e}\right\}_{e \in F}$ satisfy an additional property.

This property is motivated by the following intuition: If terminals in group $g$ witness some edges that are not in $T_{g}$, it might be cheaper to connect the terminals of $g$ in $F_{-g}$ by using those edges instead of some edges in $T_{g} \backslash F_{-g}$. If these alternate edges do not provide a cheaper connection than the corresponding edges in $T_{g} \backslash F_{-g}$, then they contribute some significant cost share to $g$ that $g$ can then use to pay for edges in $T_{g} \backslash F_{-g}$.

PROPERTY 2. There exists an order $\prec$ on the groups of terminals in $R$ such that for any two terminal groups $g, h \in R, h \prec g$ implies that all edges e of $T_{h} \backslash T_{g}$ with $\mathscr{W}_{e} \cap g=\emptyset$ are part of the forest $F_{-g}$.

While we will show in Section 3.3 that our witness definition satisfies Property 2 for groups of arbitrary size, the asymmetric cost sharing rule below is designed specifically for the case when all groups are pairs. Thus the remainder of this section is devoted to the case when all groups consist of just two terminals. In this case, we specialize our notation as follows: $P_{s t}$ is the unique path in $F$ connecting $s$ and $t$. For terminal $u$, let $\bar{u}$ be the terminal it is paired with, i.e., $(u, \bar{u}) \in R$. The cost share of a terminal pair $(u, \bar{u}) \in R$ is denoted $\xi_{u \bar{u}}$.

We define an asymmetric cost sharing rule as follows:

Asymmetric cost share assignment: Consider an edge $e \in F$ and let $\mathscr{W}_{e}=\{u, v\}$. Assume without loss of generality that $(u, \bar{u}) \prec(v, \bar{v})$. We share $\xi(e)$ among the two witnesses $u$ and $v$ as follows:

$$
\begin{aligned}
& \xi_{u}(e)=\left\{\begin{array}{ll}
\frac{1}{3} \xi(e) & \text { if } e \notin P_{u \bar{u}} \\
\frac{2}{3} \xi(e) & \text { if } e \in P_{u \bar{u}}
\end{array}\right. \text { and } \\
& \xi_{v}(e)= \begin{cases}\frac{2}{3} \xi(e) & \text { if } e \notin P_{u \bar{u}} \\
\frac{1}{3} \xi(e) & \text { if } e \in P_{u \bar{u}} .\end{cases}
\end{aligned}
$$




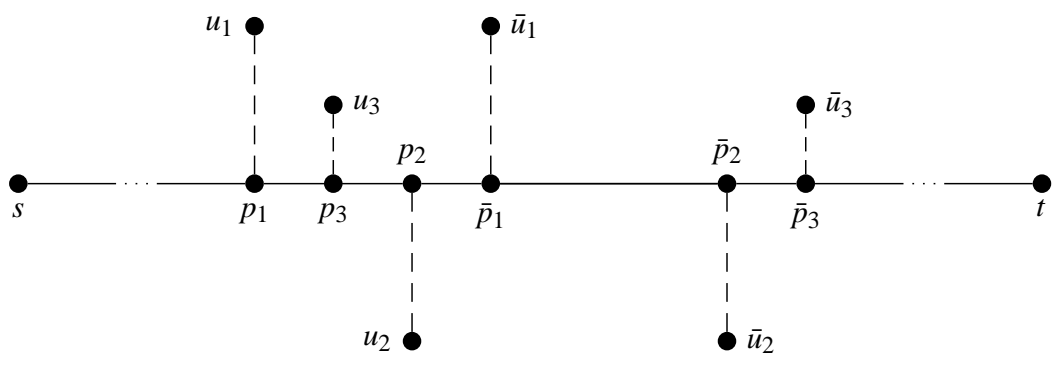

Figure 1: The figure shows the path $P_{s t}$ and three terminal pairs $\left(u_{i}, \bar{u}_{i}\right),\left(u_{j}, \bar{u}_{j}\right) \in I_{s t}$ with $1 \leq i<j \leq q$.

Observe that with this cost sharing rule, the total cost share that the witnesses in $\mathscr{W}_{e}$ receive for $e \in F$ is at $\operatorname{most} \xi(e)$.

LEMMA 3. Let $F$ be a Steiner forest computed by an $\alpha$ approximate algorithm $\mathrm{ALG}$ and let $\left\{\mathscr{W}_{e}\right\}_{e \in F}$ be the witness set. If algorithm ALG and $\left\{\mathscr{W}_{e}\right\}_{e \in F}$ satisfy Properties 1 and 2 , then the asymmetric cost shares $\xi$ are $\beta=\frac{3}{2} \alpha$-strict, i.e., for all $(s, t) \in R$

$$
c_{G \mid F_{-s t}}(s, t) \leq \frac{3}{2} \alpha \cdot \xi_{s t} .
$$

Proof. Consider path $P_{s t}$ in $F$. From Property 1 we know that each edge $e \in P_{s t}$ that is missing in $F_{-s t}$ is witnessed by at least one of $s$ and $t$. If the cost share of terminal pair $(s, t)$ for such an edge $e$ is at least $\frac{2}{3} \xi(e)$, then $\frac{3}{2} \alpha \cdot \xi_{s t}(e)$ suffices to reconstruct $e$. We can therefore reconstruct all such edges using $\frac{3}{2} \alpha$ times their total cost share. By contracting all such edges in $F_{-s t}$, we can assume without loss of generality that if $s$ or $t$ witness an edge $e \notin F_{-s t}$, then $\xi_{s t}(e)=\frac{1}{3} \xi(e)$. Let $\mathscr{M}_{s t}$ be the set of all such edges.

$$
\mathscr{M}_{s t}=\left\{e \in G \mid F_{-s t}: \xi_{s t}(e)=\frac{1}{3} \xi(e)\right\}
$$

We show that we can establish connection between $s$ and $t$ in the contracted graph at a cost at most $\frac{3}{2} \alpha \sum_{e \in \mathscr{M}_{s t}} \xi_{s t}(e)$. By then uncontracting the edges for which $\xi_{s t}(e) \geq \frac{2}{3} \xi(e)$, we have established a connection between $s$ and $t$ in $G \mid F_{-s t}$ at cost at most $\frac{3}{2} \alpha \xi_{s t}$.

By Property 2 and the cost share assignment given in (3), if $e \in$ $P_{s t} \cap \mathscr{M}_{s t}$ then the following must hold: (i) there is a terminal $u \notin$ $\{s, t\}$ that together with $s$ or $t$ witnesses $e$, (ii) $(u, \bar{u}) \prec(s, t)$ and (iii) $e \in P_{u \bar{u}}$. For these edges we need to collect additional cost share from edges in $P_{u \bar{u}} \backslash P_{s t}$ witnessed by $(s, t)$ and possibly exploit the connectivity provided by $P_{u \bar{u}}$ in $F_{-s t}$.

Let $I_{s t}$ be the set of terminal pairs that witness edges on $P_{s t}$ together with one of $s$ and $t$ :

$$
\begin{aligned}
& I_{s t}=\left\{(u, \bar{u}) \in R: \exists e \in P_{s t}\right. \text { such that } \\
&\left.\{s, t\} \cap \mathscr{W}_{e} \neq \emptyset \text { and }\{u, \bar{u}\} \cap \mathscr{W}_{e} \neq \emptyset\right\} .
\end{aligned}
$$

Assume that $I_{s t}=\left\{\left(u_{1}, \bar{u}_{1}\right), \ldots,\left(u_{q}, \bar{u}_{q}\right)\right\}$ for some $q \geq 0$. We use $P_{i}, 1 \leq i \leq q$, to refer to the $u_{i}, \bar{u}_{i}$-path in $F$. For $1 \leq i \leq q$, let $p_{i}$ and $\bar{p}_{i}$ be the first and last vertices on $P_{i}$ that are on $P_{s t}$. We call $p_{i}$ and $\bar{p}_{i}$ the projections of $u_{i}$ and $\bar{u}_{i}$, respectively, on path $P_{s t}$. We choose the label $p_{i}$ so that it is closer to $s$ than $\bar{p}_{i}$ for all $1 \leq i \leq q$. We order the indices of pairs in $I_{s t}$ so that $\bar{p}_{i}$ is closer to $s$ than $\bar{p}_{j}$ for all $1 \leq i<j \leq q$. Refer to Figure 1 for an example.

In the following, we let $P_{s t}^{i}$ be the $s, \bar{p}_{i}$-segment of $P_{s t}$ for all $0 \leq i \leq q$ (where we define $\bar{p}_{0}=s$ ). We then define

$$
\mathscr{P}^{i}=P_{s t}^{i} \cup P_{1} \cup \ldots \cup P_{i}
$$

as the union of path $P_{s t}^{i}$ and the paths of the first $i$ terminal pairs in $I_{s t}$. Finally, let $\mathscr{M}_{s t}^{i}=\mathscr{M}_{s t} \cap \mathscr{P}^{i}$ be the set of edges in $\mathscr{P}^{i}$ that are witnessed by $s$ or $t$.
For an edge set $S \subseteq \mathscr{M}_{s t}$, let $\xi_{s t}(S)$ be a short-hand for $\sum_{e \in S} \xi_{s t}(e)$. Using induction over $0 \leq i \leq q$, we will now prove that we can reconstruct an $s, z$-path in $F_{-s t}$ for all vertices $z \in \mathscr{P}^{i}$ at a cost at most $\frac{3}{2} \alpha \cdot \xi_{s t}\left(\mathscr{M}_{s t}^{i}\right)$.

This is clearly true for $i=0$. Now assume that the claim holds for some $0 \leq i<q$. Consider first the case where $\bar{p}_{i}$ precedes $p_{i+1}$ on $P_{s t}$ (see also Figure 2.(i)). Let $z$ be a node on the $\bar{p}_{i}, p_{i+1}$-segment $P$ of $P_{s t}$. Observe that none of the edges $e \in P$ can be contained on $P_{j}$ for any $1 \leq j \leq q$. Hence, if $s$ or $t$ witnessed this edge, they would receive at least a cost share of $\frac{2}{3} \xi(e)$. We excluded those edges initially and hence, using the inductive hypothesis, we can reconstruct an $s, z$-path in $F_{-s t}$ at a cost at most $\frac{3}{2} \alpha \cdot \xi_{s t}\left(\mathscr{M}_{s t}^{i}\right)$.

For the above argument, we will now assume that $p_{i+1}$ precedes $\bar{p}_{i}$ on $P_{s t}$. Let $f$ and $l$ be the first and last vertices on $P_{i+1}$, respectively, that are incident to edges of $\mathscr{P}^{i}$ (refer to Figure 2.(ii)). Clearly, the unique $f, l$-path $P_{f l}$ in $F$ must be contained in $\mathscr{P}^{i}$. Let $z_{1}$ be a node on $P_{f l}$. We can then use the induction hypothesis in order to reconstruct an $s, z_{1}$-path in $F_{-s t}$ at a cost at most $\frac{3}{2} \alpha \cdot \xi_{s t}\left(\mathscr{M}_{s t}^{i}\right)$.

Now consider a node $z_{2} \in P_{i+1} \backslash P_{f l}$. Assume that $z_{2}$ is contained on the $l, \bar{u}_{i+1}$-segment of $P_{i+1}$ (the case where $z_{2}$ is contained on the $u_{i+1}, f$-segment of $P_{i+1}$ works analogously). We consider two ways to reconstruct an $s, z_{2}$-path in $F_{-s t}$ :

1. Use $P_{f}$ for the union of the $u_{i+1}, f$ - and $z_{2}, \bar{u}_{i+1}$-segments of $P_{i+1}$, and let $\mathscr{M}_{f}$ be the set of edges on $P_{f}$ that are witnessed by $s$ or $t$. We can then inductively reconstruct an $s, f$-path in $F_{-s t}$ at a cost at most $\frac{3}{2} \alpha \cdot \xi\left(\mathscr{M}_{s t}^{i}\right)$. Using the fact that $u_{i+1}$ and $\bar{u}_{i+1}$ are connected in $F_{-s t}$, we obtain an $s, z_{2}$-path by reconstructing $P_{f}$. This costs at most $3 \alpha \cdot \xi_{s t}\left(\mathscr{M}_{f}\right)$.

2. Use $P_{l}$ for the $l, z_{2}$-segment of $P_{i+1}$ and let $\mathscr{M}_{l}$ be a shorthand for $\mathscr{M}_{s t} \cap P_{l}$. We can then inductively reconstruct an $s, l$-path in $F_{-s t}$ at a cost at most $\frac{3}{2} \alpha \cdot \xi\left(\mathscr{M}_{s t}^{i}\right)$. We obtain an $s, z_{2}$-path by reconstructing $P_{l}$ at a cost at most $3 \alpha \cdot \xi_{s t}\left(\mathscr{M}_{l}\right)$.

In summary, reconstructing an $s, z_{2}$-path in $F_{-s t}$ costs at most

$$
\begin{aligned}
& \frac{3}{2} \alpha \cdot \xi_{s t}\left(\mathscr{M}_{s t}^{i}\right)+3 \alpha \cdot \min \left\{\xi_{s t}\left(\mathscr{M}_{f}\right), \xi_{s t}\left(\mathscr{M}_{l}\right)\right\} \\
& \leq \leq \frac{3}{2} \alpha \cdot\left(\xi_{s t}\left(\mathscr{M}_{s t}^{i}\right)+\xi_{s t}\left(\mathscr{M}_{f}\right)+\xi_{s t}\left(\mathscr{M}_{l}\right)\right) .
\end{aligned}
$$

Observing that $\mathscr{M}_{f} \cup \mathscr{M}_{l} \cup \mathscr{M}_{s t}^{i}$ is a partition of $\mathscr{M}_{s t}^{i+1}$ finishes the proof of the lemma.

\section{A PRIMAL-DUAL BASED STRICT AL- GORITHM FOR STEINER FORESTS}

In this section we review a $(2-1 / k)$-approximate primal-dual algorithm for Steiner forests. The algorithms for Steiner forest presented in [3] and [15] differ only slightly. In this paper, we focus on 


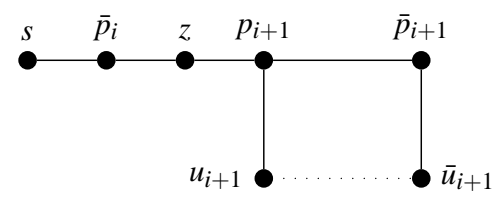

(i)

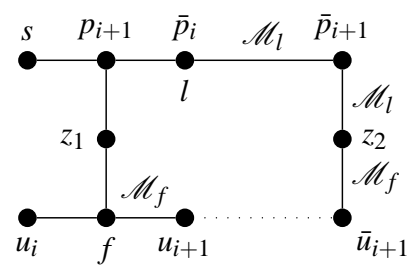

(ii)

Figure 2: The figure illustrates the cases used in the proof of Lemma 3. Solid edges represent segments of $P_{s t}$, dotted edges represent connectivity in $F_{-s t}$.

the viewpoint taken in [3]. We use AKR to refer to this algorithm. We then show that AKR together with an appropriate witness definition satisfies Properties 1 and 2.

While the Steiner forest problem is traditionally defined on pairs of nodes, it is easy to extend the definition to groups of nodes. However, in the case of the Steiner forest problem, the group problem can be modeled and solved as the problem defined on pairs, by creating a pair for each pair of nodes in a group.

\subsection{Primal-Dual Algorithms for Steiner Forests}

The primal-dual algorithm AKR constructs both a feasible primal and a feasible dual solution for a linear programming formulation of the Steiner forest problem and its dual, respectively. A standard integer programming formulation for the Steiner forest problem has a binary variable $x_{e}$ for all edges $e \in E$. Variable $x_{e}$ has value 1 if edge $e$ is part of the resulting forest. We let $\mathscr{U}$ contain exactly those subsets $U$ of $V$ that separate at least one terminal pair in $R$. In other words, $U \in \mathscr{U}$ iff there is $(s, t) \in R$ with $|\{s, t\} \cap U|=1$.

For a subset $U$ of the nodes we also let $\delta(U)$ denote the set of those edges that have exactly one endpoint in $U$. We then obtain the following integer linear programming formulation for the Steiner forest problem:

$$
\begin{array}{ll}
\min & \sum_{e \in E} c_{e} \cdot x_{e} \\
\text { s.t. } & \sum_{e \in \delta(U)} x_{e} \geq 1 \quad \forall U \in \mathscr{U} \\
& x \text { integer }
\end{array}
$$

The linear programming dual of the LP-relaxation (LP) of (IP) has a variable $y_{U}$ for all node sets $U \in \mathscr{U}$. There is a constraint for each edge $e \in E$ that limits the total dual assigned to sets $U \in \mathscr{U}$ that contain exactly one endpoint of $e$ to be at most $c_{e}$.

$$
\begin{array}{ll}
\max & \sum_{U \in \mathscr{U}} y_{U} \\
\text { s.t. } & \sum_{U \in \mathscr{U}: e \in \delta(U)} y_{U} \leq c_{e} \quad \forall e \in E \\
& y \geq 0
\end{array}
$$

Algorithm AKR constructs a primal solution for (LP) and a dual solution for (D). The algorithm has two goals:

1. Compute a feasible solution for the given Steiner forest instance. The algorithm reduces the degree of infeasibility as it progresses.

2. Create a dual feasible packing of sets of largest possible total value. The algorithm raises dual variables of certain subsets of nodes at all times. The final dual solution is maximal in the sense that no single set can be raised without violating a constraint of type (4).

Consider the execution of algorithm AKR as a process over time and let $x^{\tau}$ and $y^{\tau}$ be the primal incidence vector and feasible dual solution at time $\tau$. Note that in any optimal solution to (IP), $x_{e} \in$ $\{0,1\}$. Let $F^{\tau}$ denote the forest corresponding to the set of edges with $x_{e}^{\tau}=1$. Initially, let $x_{e}^{0}=0$ for all $e \in E$ and $y_{U}^{0}=0$ for all $U \in \mathscr{U}$. The algorithm maintains the invariant $x_{e}^{\tau} \leq x_{e}^{\tau^{\prime}}$ and $y_{U}^{\tau} \leq y_{U}^{\tau^{\prime}}$ for all $\tau<\tau^{\prime}$.

An edge $e \in E$ is tight if the corresponding constraint (4) holds with equality; and a path is tight if every edge in the path is tight. Assume that the forest $F^{\tau}$ at time $\tau$ is infeasible. A terminal node $v \in R$ is active at time $\tau$ if $v$ and its mate $\bar{v}$, i.e., $(v, \bar{v}) \in R$, are in different trees in the forest $F^{\tau} ; v$ is inactive otherwise. ${ }^{1}$ Let $\bar{F}^{\tau}$ denote the subgraph of $G$ that is induced by the tight edges for dual $y^{\tau}$. To avoid confusion between connected components in $F^{\tau}$ and those in $\bar{F}^{\tau}$, the term moat refers to a connected component in $\bar{F}^{\tau}$. The algorithm maintains that if $C \in F^{\tau}$ then $C \subseteq U \in \bar{F}^{\tau}$. A moat $U$ of $\bar{F}^{\tau}$ is active at time $\tau$ if $U$ contains an active terminal; $U$ is inactive otherwise. Let $A^{\tau}$ be the set of all active moats in $\bar{F}^{\tau}$ at time $\tau$. AKR raises the dual variables for all sets in $A^{\tau}$ uniformly at all times $\tau \geq 0$, so that if $U$ is active from time $\tau^{\prime}$ until time $\tau^{\prime \prime}$, then $y_{U}=\tau^{\prime \prime}-\tau^{\prime}$.

Two disjoint moats collide at time $\tau$ in the execution of AKR if there is a path in $G$ from one moat to the other that becomes tight at time $\tau$. In order for this to happen, at least one of the two moats must be active. Suppose that a path $P$ connecting two active terminals $u$ and $u^{\prime}$ becomes tight at time $\tau$ in the execution of AKR. Then $u$ is contained in some active moat $U$ and $u^{\prime}$ is in a disjoint active moat $U^{\prime}$. When this happens, AKR adds the edges of $P$ not already in $F^{\tau}$ to $F^{\tau}$ : that is, for all $e \in P$, the algorithm sets $x_{e}^{\tau}=1$. For $\tau^{\prime}>\tau$, sets $U$ and $U^{\prime}$ are part of the same moat of $\bar{F}^{\tau^{\prime}}$.

Subsequently, we use $U^{\tau}(v)$ to refer to the moat in $\bar{F}^{\tau}$ that contains node $v \in V$ at time $\tau$. Similarly, we let $U^{\tau}(C)$ denote the moat in $\bar{F}^{\tau}$ that contains the connected component $C \in F^{\tau}$ at time $\tau$. Let $F$ be the final forest.

The following is the main theorem of [3]:

THEOREM 2. Let $F$ be the forest computed by AKR on terminal set $R$. We then have $c(F) \leq\left(2-\frac{1}{k}\right) \cdot \mathrm{opt}_{R}$, where $\mathrm{opt}_{R}$ is the minimum cost of a Steiner forest for the given input instance with terminal set $R$.

\subsection{Witness Definition}

We define a set $\left\{\mathscr{W}_{e}\right\}_{e \in F}$ of witnesses that are used to distribute the cost shares as described in Section 2. In Section 3.3 we show

${ }^{1}$ Note that for the problem defined on groups, each terminal in the group will become inactive at exactly the same time, since if the group is not connected, then each terminal is not connected to some other terminal in the group. 


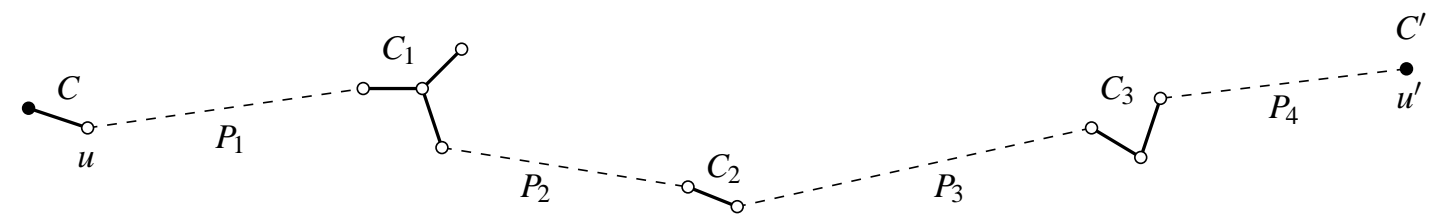

Figure 3: A path $P$ that becomes tight at time $\tau_{P}$ in $\operatorname{AKR}(R)$.

that AKR together with this witness definition satisfies Properties 1 and 2.

Let $\mathrm{AKR}(S)$ refer to the execution of AKR on terminal set $S \subseteq R$. Let $F$ be the forest computed by $\operatorname{AKR}(R)$ for terminal set $R$. The witnesses $\mathscr{W}_{e}$ for each edge $e \in F$ are defined by the execution of $\operatorname{AKR}(R)$.

Consider a path $P$ that becomes tight at time $\tau_{P}$ in $\operatorname{AKR}(R)$, as depicted in Figure 3. Path $P$ starts from a node $u$ in a connected component $C$ of $F^{\tau_{P}}$, passes through a (possibly empty) sequence $C_{1}, \ldots, C_{l}$ of connected components of $F^{\tau_{P}}$, and ends in a node $u^{\prime}$ of a connected component $C^{\prime}$ of $F^{\tau_{P}}$. Let $P_{1}, \ldots, P_{l+1}$ be the sequence of paths of $P \backslash F^{\tau_{P}}$ and let $\mathscr{P}_{P}$ be the set of edges in $P \backslash F^{\tau_{P}}$. When $P$ becomes tight, the set $\mathscr{P}_{P}$ is added to $F$ and we determine for each edge $e \in \mathscr{P}_{P}$ the corresponding witnesses $\mathscr{W}_{e}$ as follows.

Each edge $e \in \mathscr{P}_{P}$ will have the same witness set $\mathscr{W}_{e}=\left\{w, w^{\prime}\right\}$. We will also say that $P$ is witnessed by $w$ and $w^{\prime}$. Since the moats $U^{\tau_{P}}(C)$ and $U^{\tau_{P}}\left(C^{\prime}\right)$ are active at time $\tau_{P}$, both $C$ and $C^{\prime}$ must contain at least one active terminal. We will choose one witness among the active terminals in each of $C$ and $C^{\prime}$. Intuitively, the witness chosen in $C$ is the active terminal whose moat intersects $P_{1}$ earliest among all active terminals in $C$. Similarly, the moat of the witness chosen in $C^{\prime}$ is the first to intersect $P_{l+1}$ among all active terminals in $C^{\prime}$. To make this precise, let $A_{C}$ be the set of terminals in $C$ that are active at time $\tau_{P}$. By definition of $C$, all terminals in $A_{C}$ are connected to $u$ in $F^{\tau_{P}}$. If $u \in A_{C}$, then $w=u$. Otherwise, let $\tau_{u}$ be the first time that moat $U^{\tau}(u)$ collides with a moat $U_{u}$ containing a terminal in $A_{C}$. (Let $\tau_{u}=0$ if $u \in A_{C}$.)

LEMMA 4. There is a terminal $w$ in $U_{u} \cap A_{C}$ whose moat collides with u's moat at time $\tau_{u}$ even if all terminals in $A_{C} \backslash\{w\}$ are not part of the terminal set $R$.

PROOF. We will prove the lemma by showing a stronger claim: For all terminals $v$ in $C$ that become inactive before time $\tau_{P}$, let $\tau_{v}$ be the first time that moat $U^{\tau}(v)$ collides with a moat $U_{v}$ containing at least one terminal from $A_{C}$. Then there is a terminal $w \in U_{v} \cap A_{C}$ whose moat collides with $v$ 's moat at time $\tau_{v}$ even if all terminals in $A_{C} \backslash\{w\}$ are not part of the terminal set $R$. This clearly implies the lemma.

Fix a terminal $v$ in $C$ that becomes inactive at some time before $\tau_{P}$. Observe that by the definition of $\tau_{v}, U^{\tau}(v)$ does not intersect $A_{C}$ before time $\tau_{v}$, and therefore the growth of $v$ 's moat until time $\tau_{v}$ is not affected by the removal of $A_{C}$.

The proof is by induction on $\left|U_{v}\right|$. If $\left|U_{v}\right|=1$ then the set consists of only a terminal $w \in A_{C}$, and the growth of $w$ 's moat is not affected by the removal of other terminals in $A_{C}$.

Now assume that $\left|U_{v}\right|>1$. Let $z \in U_{v}$ be the endpoint of the path $P_{v}$ that becomes tight when $U_{v}$ collides with $U^{\tau}(v)$. If $z$ is in $A_{C}$ we are done: We define $w=z$ and observe that $w$ 's moat intersects $P_{v}$ at all times $0 \leq \tau \leq \tau_{v}$ even if the terminals in $A_{C} \backslash\{w\}$ are not part of the terminal set.

Assume that $z$ is not in $A_{C}$. In this case, let $\tau_{z}$ be the first time that $U^{\tau}(z)$ collides with a moat $U_{z}$ that contains a terminal from $A_{C}$. We have $\left|U_{z}\right|<\left|U_{v}\right|$ and can therefore apply the induction hypothesis to $z$ and $U_{z}$. That is, there is a terminal $w \in U_{z}$ whose moat collides with $z$ 's moat at time $\tau_{z}$ even if all terminals in $A_{C} \backslash\{w\}$ are not part of the terminal set $R$. Since $w$ is in $A_{C}$, it causes the moat containing $z$ to grow after time $\tau_{z}$ regardless of other terminals in $A_{C}$. Thus, $w$ 's moat collides with that of $v$ at time $\tau_{v}$ and this finishes the lemma.

The witness $w$ is a terminal described by Lemma 4 . The witness $w^{\prime}$ with respect to $C^{\prime}$ is defined analogously.

\subsection{Properties of $A K R$}

We show that Properties 1 and 2 hold for AKR and the witness definition given above. Let $\left\{\mathscr{W}_{e}\right\}_{e \in F}$ be the set of witnesses assigned by AKR. Let $\mathscr{G}_{-g}$ (where $\mathscr{G}=U, F$ or $\bar{F}$ ) refer to set $\mathscr{G}$ in run $\operatorname{AKR}\left(R_{-g}\right)$. For example, $U_{-g}^{\tau}(u)$ refers to the moat of $u$ at time $\tau$ in $\operatorname{AKR}\left(R_{-g}\right)$. Let $\tau_{g}$ denote the time at which all terminals in group $g$ become inactive in $\operatorname{AKR}(R)$. Subsequently, we abuse notation by letting $R$ also refer to the set of all terminals that are contained in the groups of $R$.

LeMma 5. For all $\tau \leq \tau_{g}$ and for all terminals $v \in R_{-g}$ : $U_{-g}^{\tau}(v) \subseteq U^{\tau}(v)$. Moreover, if $U^{\tau}(v) \cap g=\emptyset$, then $U_{-g}^{\tau}(v)=U^{\tau}(v)$.

Proof. We prove the lemma by induction over time $\tau$. At time $\tau=0$ we have $U_{-g}^{\tau}(v)=U^{\tau}(v)$ for all $v \in R_{-g}$ and thus the induction hypothesis clearly holds. Assume the induction hypothesis holds at time $\tau$. We will show that it remains true at time $\tau+\varepsilon$ for any small $\varepsilon>0$.

Consider the case $U^{\tau}(v) \cap g=\emptyset$ and thus $U_{-g}^{\tau}(v)=U^{\tau}(v)$. That is, $U_{-g}^{\tau}(v)$ is active at time $\tau$ iff $U^{\tau}(v)$ is active at that time. Then $U_{-g}^{\tau+\varepsilon}(v)=U^{\tau+\varepsilon}(v)$ if $U^{\tau+\varepsilon}(v) \cap g=\emptyset$; and $U_{-g}^{\tau+\varepsilon}(v) \subseteq U^{\tau+\varepsilon}(v)$ otherwise. Now assume $U^{\tau}(v) \cap g \neq \emptyset$ and thus $U_{-g}^{\tau}(v) \subseteq U^{\tau}(v)$. Clearly, $U^{\tau+\varepsilon}(v) \cap g \neq \emptyset$. Since $\tau \leq \tau_{g}$, all terminals in $g$ are active at time $\tau$ and thus $U^{\tau}(v)$ is active at time $\tau$. It follows $U_{-g}^{\tau+\varepsilon}(v) \subseteq$ $U^{\tau+\varepsilon}(v)$.

COROLlary 1. Consider a terminal $v \in R_{-g}$. If $v$ is active at time $\tau \leq \tau_{g}$ in $\operatorname{AKR}(R)$ then $v$ must be active until time at least $\tau$ in $\operatorname{AKR}\left(R_{-g}\right)$.

As in Figure 3, let $P$ be a path connecting two active components $C$ and $C^{\prime}$ that becomes tight at time $\tau_{P} \leq \tau_{g}$ in the execution of $\operatorname{AKR}(R)$. As before, let $u$ and $u^{\prime}$ be the two endpoints in $C$ and $C^{\prime}$, respectively, and let $C_{1}, \ldots, C_{l}$ be the connected components of $F^{\tau_{P}}$ that lie on $P$. Moreover, assume $P$ is witnessed by $w$ and $w^{\prime}$. The following lemma implies Property 1 for our definition of witnesses.

Lemma 6. Assume that none of the two witnesses $w, w^{\prime}$ of $P$ is in $g$, i.e., $\mathscr{W}_{e} \cap g=\emptyset$ for all edges $e \in \mathscr{P}_{P}$. Then for each edge $e \in P$, the contribution to (4) before time $\tau_{P}$ is the same in $\operatorname{AKR}(R)$ as it is in $\operatorname{AKR}\left(R_{-g}\right)$. In particular, $P$ is added at time $\tau_{P}$ in both runs.

PROOF. First we show that the contribution to (4) from variables corresponding to moats not containing $u$ and $u^{\prime}$ is the same in both runs. For all $1 \leq i \leq l, C_{i}$ is inactive at time $\tau_{P}<\tau_{g}$, so that $C_{i} \cap g=$ 


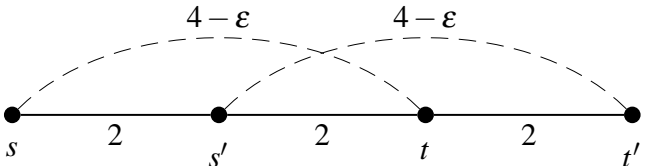

(i)

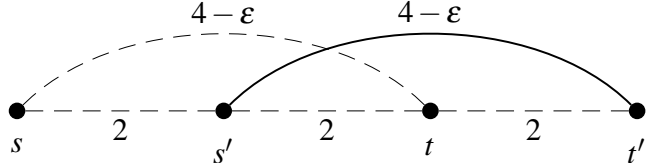

(ii)

Figure 4: Instance used in the lower bound argument.

$\emptyset$. Let $\tau_{i}<\tau_{P}$ be the time at which component $C_{i}$ becomes inactive. Then, by Lemma $5, U_{g}^{\tau}(v)=U^{\tau}(v)$ for all $\tau \leq \tau_{i}$, for all $v \in C_{i}$, for all $1 \leq i \leq l$. Thus, the dual variable values for all sets restricted to subsets of $C_{i}$ are the same in both $\operatorname{AKR}(R)$ and $\operatorname{AKR}\left(R_{-g}\right)$.

Now consider the contribution to (4) from variables corresponding to moats containing $u$. Let $\hat{\tau}$ be the first time at which moat $U^{\hat{\tau}}(u)$ collides with a moat $\hat{U}$ containing a terminal in $A_{C}$. By the definition of $\hat{\tau}, U^{\tau}(u) \cap g=\emptyset$ for all $\tau \in[0, \hat{\tau})$. Thus by Lemma 5, $U_{-g}^{\tau}(u)=U^{\tau}(u)$ for all $\tau \in[0, \hat{\tau})$; and the contribution to (4) from variables corresponding to moats containing $u$ before time $\hat{\tau}$ is the same in $\operatorname{AKR}(R)$ and $\operatorname{AKR}\left(R_{-g}\right)$. From time $\hat{\tau}, w$ and $u$ are in the same moat in $\operatorname{AKR}(R)$, and by Lemma 4, they are also in the same moat at this time in $\operatorname{AKR}\left(R_{-g}\right)$. By Lemma $5, w$ is still active at time $\tau_{P}$ in both $\operatorname{AKR}(R)$ and $\operatorname{AKR}\left(R_{-g}\right)$. Thus the contribution to (4) of variables corresponding to moats containing $u$ from time $\hat{\tau}$ until time $\tau_{P}$ is also the same in both runs. A symmetric argument for variables corresponding to moats containing $u^{\prime}$ shows that path $P$ is tight at time $\tau_{P}$ in $\operatorname{AKR}\left(R_{-s t}\right)$.

Finally, note that Lemma 5 also implies that $w$ and $w^{\prime}$ are contained in disjoint moats in $\operatorname{AKR}\left(R_{-s t}\right)$ before time $\tau_{P}$. Hence path $P$ is added at time $\tau_{P}$ in $\operatorname{AKR}\left(R_{-s t}\right)$ and the lemma follows.

We show that the following precedence order $\prec$ together with the witness definition described above imply Property 2 for AKR.

Consider a run $\operatorname{AKR}(R)$. For each group of terminals $g \in R$, let $\tau_{g}$ be the time at which the terminals in $g$ become inactive. Fix an order on the terminal groups in $R=\left\{g_{i}\right\}_{1 \leq i \leq k}$ such that

$$
\tau_{g_{1}} \leq \tau_{g_{2}} \leq \cdots \leq \tau_{g_{k}} .
$$

We define $g_{i} \prec g_{j}$ if $i \leq j$ in this order.

The following lemma implies Property 2 .

LEMMA 7. Let $g$ and $h$ be two groups of terminals in $R$ such that $h \prec g$ and let e be an edge on tree $T_{h}$ in $F$. If $\mathscr{W}_{e} \cap g=\emptyset$ then $e \in F_{-g}$.

PROOF. The proof is by contradiction. Assume that edge $e$ is not part of $F_{-g}$. Edge $e \in T_{h}$ is added to $F$ at time $\tau \leq \tau_{h} \leq \tau_{g}$. By Lemma 6 and since $\mathscr{W}_{e} \cap g=\emptyset, e$ is picked at time $\tau$ in $\operatorname{AKR}\left(R_{-g}\right)$. This is a contradiction.

\section{A LOWER BOUND ON THE STRICT- NESS FACTOR}

Figure 4 shows a simple Steiner forest instance with two terminal pairs $R=\left\{(s, t),\left(s^{\prime}, t^{\prime}\right)\right\}$. The solid lines in Figure 4.(i) correspond to edges of forest $F$ returned by algorithm AKR when run on this instance. The total cost share of all edges in $F$ is 3 and therefore, there must be a terminal pair in $R$ whose cost share is at most $\frac{3}{2}$. Without loss of generality, assume that $\xi_{s t} \leq \frac{3}{2}$. Running AKR on terminal set $R_{-s t}=\left\{\left(s^{\prime}, t^{\prime}\right)\right\}$ yields the forest in Figure 4.(ii). As $c_{G \mid F_{-s t}}(s, t)=4-\varepsilon$, this example shows that the strictness of AKR is at least $(4-\varepsilon) / \frac{3}{2} \approx \frac{8}{3}$ whenever the sum of the cost shares of all terminal pairs is at most half of the cost of the computed forest.
We remark that the previously known algorithms for the MRoB problem in [10] and [6] essentially distribute half of the cost of a forest computed by AKR as cost shares among the terminal pairs. Given a terminal pair $(s, t) \in R$, both of these algorithms use an adaptation of the standard primal-dual Steiner forest algorithm (so called timed or boosted primal-dual algorithms) to compute a forest $F_{-s t}$. In a nutshell, the idea behind these adaptations is to produce a forest whose connectivity is higher than that of a forest produced by standard primal-dual algorithms. For the example above, however, both algorithms in [10] and [6] return the forest in Figure 4.(ii). Thus, the above example provides an $\frac{8}{3}$ lower bound for the strictness of these algorithms as well.

\section{REFERENCES}

[1] M. Andrews. Hardness of buy-at-bulk network design. In Proceedings, IEEE Symposium on Foundations of Computer Science, pages 115-124, 2004.

[2] B. Awerbuch and Y. Azar. Buy-at-bulk network design. In Proceedings, IEEE Symposium on Foundations of Computer Science, pages 542-547, 1997.

[3] A. Agrawal, P. Klein, and R. Ravi. When trees collide: An approximation algorithm for the generalized Steiner problem in networks. SIAM J. Comput., 24:440-456, 1995.

[4] Y. Bartal. Probabilistic Approximation of Metric Spaces and its Algorithmic Applications. In Proceedings, IEEE Symposium on Foundations of Computer Science, pages 184-193, 1996.

[5] Y. Bartal. On approximating arbitrary metrics by tree metrics. In Proceedings, ACM Symposium on Theory of Computing, pages 161-168, 1998.

[6] L. Becchetti, J. Könemann, S. Leonardi, and M. Pál. Sharing the cost more efficiently: Improved approximation for multicommodity rent-or-buy. In Proceedings, ACM-SIAM Symposium on Discrete Algorithms, pages 375-384, 2005.

[7] M. Bern and P. Plassmann. The Steiner problem with edge lengths 1 and 2. Inform. Process. Lett., 32(4):171-176, 1989.

[8] J. Fakcharoenphol, S. Rao and K. Talwar. A tight bound on approximating arbitrary metrics by tree metrics. In Proceedings, ACM Symposium on Theory of Computing, pages 448-455, 2003.

[9] M. R. Garey and D. S. Johnson. Computers and Intractability: A Guide to the Theory of NP-completeness. Freeman, San Francisco, 1979.

[10] A. Gupta, A. Kumar, M. Pál, and T. Roughgarden. Approximation via cost-sharing: A simple approximation algorithm for the multicommodity rent-or-buy problem. In Proceedings, IEEE Symposium on Foundations of Computer Science, pages 606-617, 2003.

[11] A. Gupta, A. Kumar, and T. Roughgarden. Simpler and better approximation algorithms for network design. In Proceedings, ACM Symposium on Theory of Computing, pages 365-372, 2003. 
[12] A. Gupta and M. Pál. Stochastic Steiner trees without a root. In Proceedings, International Colloquium on Automata, Languages and Programming, pages 1051-1063, 2005.

[13] A. Gupta, M. Pál, R. Ravi, and A. Sinha. Boosted sampling: Approximation algorithms for stochastic optimization. In Proceedings, ACM Symposium on Theory of Computing, pages 417-426, 2004.
[14] A. Gupta, R. Ravi, and A. Sinha. An edge in time saves nine: LP rounding approximation algorithms. In Proceedings, IEEE Symposium on Foundations of Computer Science, pages 218-227, 2004.

[15] M. X. Goemans and D. P. Williamson. A general approximation technique for constrained forest problems. SIAM J. Comput., 24:296-317, 1995.

[16] A. Kumar, A. Gupta, and T. Roughgarden. A constant factor approximation algorithm for the multicommodity rent-or-buy problem. In Proceedings, IEEE Symposium on Foundations of Computer Science, pages 333-344, 2002. 